\title{
BALANCE PARCIAL DE NITRÓGENO EN EL SISTEMA DE CULTIVO DE MAÍZ (Zea mays L.) CON COBERTURA DE LEGUMINOSAS EN CHIAPAS, MÉXICO
}

\author{
José David Álvarez-Solís ${ }^{l *}$, Ramón Muñoz-Arroyo*, Esperanza Huerta-Lwanga*, José Nahed-Toral \\ Palabras clave: Cultivos de cobertura; fijación biológica de nitrógeno; suministro de nitrógeno; \\ leguminosas de grano. \\ Keywords: Cover crops; biological nitrogen fixation; supply of nitrogen; grain leguminous.
}

Recibido: 24/07/2015

\section{RESUMEN}

El potencial de las leguminosas para mejorar la fertilidad del suelo y sostener la producción de cultivos asociados como el maíz, varía entre especies. Este trabajo evalúa la contribución de 3 leguminosas como cultivos de cobertura (CC) en el balance parcial de Nitrógeno $(\mathrm{N})$ en el cultivo de maíz. Fue realizado en Ejido La Bella Ilusión, Maravilla Tenejapa, estado de Chiapas, México, con 4 tratamientos en asociación al cultivo de maíz: 1) frijol nescafé (Mucuna pruriens), 2) frijol arroz (Vigna umbellata), 3) frijol común (Phaseolus vulgaris) y 4) testigo sin leguminosa. Se midió la producción de biomasa y el contenido de $\mathrm{N}$, en las leguminosas y el maíz. Se hizo un balance parcial de $\mathrm{N}$, que consideró el suministro de $\mathrm{N}$ de la biomasa de leguminosas y la extracción de $\mathrm{N}$ en la cosecha del maíz. La producción de biomasa seca de las leguminosas en la cosecha fue significativamente $(\mathrm{p} \leq 0,05)$ mayor en asociación con los frijoles arroz y nescafé (1290 y 1139 $\left.\mathrm{kg} \cdot \mathrm{ha}^{-1}\right)$ que en frijol común $\left(470 \mathrm{~kg} \cdot \mathrm{ha}^{-1}\right)$, con un contenido total de 35,$4 ; 25,7$ y $8,9 \mathrm{~kg}^{-h^{-1}} \mathrm{~N}$, respectivamente. El rendimiento de grano de maíz fue $2390,5 \mathrm{~kg} . \mathrm{ha}^{-1}$; se identificó que la extracción de $\mathrm{N}$ fue mayor en el grano, seguido de rastrojo, raíz, bráctea y olote, con 20,9; 9,4; 7,2; 2,0 y 1,5 kg.ha- ${ }^{-1} \mathrm{~N}$, respectivamente. El balance parcial de

1 Autor para correspondencia. Correo electrónico: dalvarez@ecosur.mx
Aceptado: 15/12/15 
$\mathrm{N}$ mostró valores positivos con los frijoles arroz y nescafé, lo que indica su importancia para compensar la extracción de $\mathrm{N}$ del grano o de la mazorca del maíz.

\section{INTRODUCCIÓN}

Un aspecto importante a considerar en la producción agrícola, es el adecuado suministro de nutrientes para satisfacer los requerimientos de un cultivo. El maíz requiere altas cantidades de $\mathrm{N}$ para la formación del grano ya que su ausencia o insuficiencia determina una baja productividad (Mendoza et ál. 2006). La cantidad de $\mathrm{N}$ que requiere el cultivo de maíz para producir una tonelada de grano y la estructura vegetativa es de 20 a 29 kg de N (Pérez et ál. 2000, Castellanos et ál. 2005). Los cultivos de cobertura (CC) son una alternativa para mejorar la fertilidad del suelo y la productividad del cultivo (FAO 1999), debido a que incrementan el contenido de la materia orgánica, la capacidad de intercambio de cationes y la disponibilidad de macro y micro nutrimentos del suelo. También disminuyen la presencia de arvenses, plagas y enfermedades; favorecen la formación de agregados estables, mejoran la infiltración del agua e incorporan $\mathrm{N}$ al suelo través de la fijación biológica (Franke et ál. 2008, Ayala et ál. 2009, Castillo et ál. 2010).

El frijol nescafé (Mucuna pruriens (L.) D.C.), es una leguminosa usada como CC en los sistemas agrícolas de bajos insumos (CIDICCO 1991, Nyambati 2002). Esta especie tiene potencial de fijación biológica de $\mathrm{N}_{2}$ cercano a $200 \mathrm{~kg} \cdot \mathrm{ha}^{-1}$ y así proporciona cantidades altas de materia orgánica y $\mathrm{N}$ al suelo (Franke et ál. 2008) aunque su adopción por los productores en México es limitada (Buckles y Triomphe 1999), lo cual podría ser asignado al riesgo de invertir tierra, trabajo y semillas en una tecnología que no
$\mathrm{N}$, respectively. The partial balance of $\mathrm{N}$ showed positive values with "rice" and "velvet" beans, which indicates their importance in compensating the $\mathrm{N}$ extraction by corn grain or ear.

provee un retorno económico rápido y seguro o bien por no contribuir con la fijación biológica de $\mathrm{N}_{2}$ en esta especie que desciende a $20 \mathrm{~kg} \cdot \mathrm{ha}^{-1}$ de $\mathrm{N}$ en suelos infértiles o con exceso de humedad (Noordwijk et ál. 1995, Becker y Johnson 1998) e incluso podría estar ausente cuando no se inocula con el Bradyrhizobium específico (Houngnandan et ál. 2000).

Por el contrario, algunas leguminosas de grano, como el frijol común (Phaseolus vulgaris L.) y el frijol arroz (Vigna umbellata Thunb.) son componentes importantes de los sistemas agrícolas tradicionales que contribuyen con la producción de granos comestibles y que sin embargo sus beneficios en términos de la fertilidad del suelo, control de arvenses y producción del maíz no han sido suficientemente evaluados (Hungria et ál. 2003). Flores et ál. (2005) indicaron que el frijol común y el frijol arroz se caracterizan por su alto valor nutritivo y adaptación a condiciones secas aunque, el frijol arroz es utilizado como CC y ha logrado adaptarse a condiciones húmedas.

Una herramienta para analizar la contribución de las leguminosas en la fertilidad del suelo es el balance nutrimental ya que permite analizar la cantidad de nutrimentos que entran y salen de un campo de cultivo; este balance se usa como indicador del uso sostenible en sistemas agrícolas (Dechert et ál. 2005). En esta prueba se considera que la fertilidad del suelo está determinada principalmente por la relación que se establece entre los nutrimentos exportados a través de la cosecha y los incorporados a través de las prácticas de fertilización (mineral u orgánica) y se considera 
que los flujos internos entre los reservorios de nutrimentos se encuentran en equilibrio dinámico (Van der Pool 1992). De acuerdo con Dechert et ál. (2005), las prácticas de manejo que resultan en un balance desfavorable conducen al sistema agrícola hacia el uso insostenible del suelo que con el tiempo tiende a la degradación. Por lo anterior, es de esperar que además de frijol nescafé otras leguminosas utilizadas como CC aporten cantidades importantes de $\mathrm{N}$ para sustentar la producción de maíz.

\section{MATERIALES Y MÉTODOS}

\section{Área de estudio y características del suelo}

El estudio se llevó a cabo en el ciclo setiembre a febrero (otoño-invierno) 2009-2010 bajo condiciones de humedad residual o "tornamil" y prácticas de manejo locales en Ejido La Bella Ilusión del municipio de Maravilla Tenejapa, Chiapas, México; el ejido se localiza en la Región de la Selva Lacandona, a $16^{\circ} 08^{\prime} \mathrm{N}$ y $91^{\circ} 17^{\prime} \mathrm{O}$, con altitud de $400 \mathrm{~m}$ sobre el nivel medio del mar. El clima es cálido húmedo (Am); con 2000-3000 mm de precipitación anual; y suelo del tipo Luvisol crómico (IUSS 2007).

El trabajo se realizó con un grupo de productores autodenominado "La Ventana" que transita de la agricultura de Roza y Quema (RQ) hacia un sistema de milpa de Roza y Pica (RP) mediante un proceso de innovación y desarrollo local con tecnologías de producción agroecológica, entre las que se encuentran el uso de cultivos de cobertura (CC) y biofertilizantes como alternativa para sostener la producción del maíz.

Se seleccionaron 6 sitios (P1, P2, P3, P4, P5 y P6) y en cada uno de ellos se aplicó una réplica de los 4 tratamientos evaluados. Previo a la siembra se recolectó una muestra compuesta de suelo con 15 puntos de muestreo en zig-zag, a una profundidad de $20 \mathrm{~cm}$, con una barrena de cilindro de acero inoxidable y diámetro de $2,54 \mathrm{~cm}$. Las muestras compuestas de suelo fueron procesadas en el laboratorio de suelos y plantas de ECOSUR, en donde se pusieron a secar al aire, luego se molieron y tamizaron para realizar las siguientes composiciones: textura (Bouyoucus), materia orgánica (Walkley y Black), fósforo extraíble (Olsen), nitrógeno total (semi-microKjeldahl), pH (relación 1:2 con $\mathrm{H}_{2} \mathrm{O}$ ), capacidad de intercambio catiónico (acetato de amonio $1 \mathrm{~N} \mathrm{pH} \mathrm{7),} \mathrm{densidad} \mathrm{aparente} \mathrm{(probeta)} \mathrm{y}$ las bases intercambiables: potasio, calcio y magnesio (acetato de amonio 1N, pH 7) (SEMARNAT 2002). Los datos de dichas determinaciones se muestran en el Cuadro 1. Los análisis de suelo mostraron que todas las parcelas presentaron textura arcillosa y una densidad aparente cercana a la unidad, lo que indica condiciones favorables en la aireación, la porosidad y la capacidad de infiltración (Landon 1991). Asimismo, presentaron niveles muy altos de materia orgánica y de la capacidad de intercambio de cationes, y un $\mathrm{pH}$ ligeramente ácido (SEMARNAT 2002). 
Cuadro 1. Características físicas y químicas del suelo de los sitios experimentales.

\begin{tabular}{|c|c|c|c|c|c|c|c|}
\hline \multirow{2}{*}{ Características } & \multicolumn{6}{|c|}{ Sitios } & \multirow{2}{*}{ Media } \\
\hline & P1 & P2 & P3 & P4 & P5 & P6 & \\
\hline Arena $(\%)$ & 24,4 & 18,4 & 30,4 & 22,4 & 22,4 & 22,4 & 23,4 \\
\hline Limo $(\%)$ & 20,0 & 24,0 & 16,0 & 18,0 & 14,0 & 22,0 & 19,0 \\
\hline Arcilla (\%) & 55,6 & 57,6 & 53,6 & 59,6 & 63,6 & 55,6 & 57,6 \\
\hline Densidad aparente $\left(\mathrm{g} . \mathrm{cm}^{-3}\right)$ & 0,96 & 1,00 & 0,99 & 0,96 & 1,01 & 0,97 & 0,98 \\
\hline $\mathrm{pH}$ & 6,2 & 7,0 & 6,6 & 7,1 & 6,1 & 5,2 & 6,4 \\
\hline $\mathrm{MO}(\%)$ & 8,70 & 7,69 & 9,70 & 9,70 & 9,37 & 8,36 & 8,92 \\
\hline $\mathrm{N}$ total $(\%)$ & 0,52 & 0,44 & 0,55 & 0,59 & 0,55 & 0,48 & 0,52 \\
\hline P-Olsen (mg. $\mathrm{kg}^{-1}$ ) & 12,1 & 10,5 & 12,5 & 11,8 & 11,2 & 11,8 & 11,7 \\
\hline Potasio $\left(\mathrm{cmol} \cdot \mathrm{kg}^{-1}\right)$ & 0,8 & 0,8 & 0,6 & 1,7 & 0,5 & 1,5 & 1,0 \\
\hline Calcio $\left(\mathrm{cmol} \cdot \mathrm{kg}^{-1}\right)$ & 51,0 & 38,2 & 40,4 & 48,8 & 53,7 & 41,7 & 45,6 \\
\hline Magnesio (cmol. $\mathrm{kg}^{-1}$ ) & 5,2 & 5,4 & 4,4 & 5,8 & 8,2 & 6,4 & 5,9 \\
\hline CIC $\left(\mathrm{cmol} \cdot \mathrm{kg}^{-1}\right)$ & 61,2 & 57,2 & 62,1 & 65,3 & 69,3 & 61,6 & 62,8 \\
\hline
\end{tabular}

\section{Tratamientos y diseño experimental}

Los tratamientos evaluados fueron: 1) frijol nescafé (FN) (M. pruriens), 2) frijol arroz (FA) (V. umbellata), 3) frijol común (FC) (P. vulgaris), y 4) testigo sin leguminosa (T). El diseño experimental fue bloques completos distribuidos al azar con 6 repeticiones. No se incluyó un tratamiento con fertilizantes en una dosis racional debido al interés de conocer el potencial de rendimiento del maíz bajo las condiciones de manejo local. El tamaño de la unidad experimental fue en promedio de $21 \mathrm{~m}$ de largo por $11 \mathrm{~m}$ de ancho. La siembra de los cultivos se realizó de acuerdo con el conocimiento y práctica de los productores y su percepción en relación con el clima. En el caso de maíz, ésta se realizó a finales de noviembre en 3 parcelas y a principios de enero en las otras 3; la siembra tardía se debió a la necesidad de la presencia de lluvias para tener buena humedad y asegurar la germinación. Se plantaron con espeque 4-5 semillas de maíz en cada hoyo de siembra, a una distancia entre hileras y matas de 1 y $0,8 \mathrm{~m}$, respectivamente. A los 30 días después de la siembra del maíz (ddsm) se realizó la siembra de leguminosas, con espeque de 3-4 semillas entre hileras del maíz, a una distancia promedio entre matas de $0,4 \mathrm{~m}$ para $\mathrm{FC}$; 0,9 m para FN; y $0,5 \mathrm{~m}$ para FA.

La preparación del terreno se realizó mediante un "chaporréo" con machete, que consistió en rozar la vegetación y dejarla sobre el terreno. Previo a la siembra, en todos los tratamientos las semillas de maíz fueron recubiertas con raíces finamente molidas del pasto cubano (Brachiaria brizantha) con un $84 \%$ de colonización micorrízica arbuscular (Glomus sp.), propagado en la localidad y aplicado en una proporción aproximada de $0,5 \mathrm{~kg}$ del inóculo por cada $6 \mathrm{~kg}$ de semilla, con agua como adherente. Asimismo, el follaje de maíz fue asperjado en 3 ocasiones con un biofertilizante foliar, elaborado localmente a base de estiércol fresco de vaca (40 $\mathrm{kg}$ ), piloncillo o melaza (9 1), leche (9 1), ceniza de fogón $(1,5 \mathrm{~kg})$, polvo de roca $(1 \mathrm{~kg})$ y agua (100 1). La primera aplicación del fertilizante foliar se realizó cuando el maíz tuvo entre 20 a $40 \mathrm{~cm}$ de altura; la segunda cuando alcanzó una altura de 60 a $70 \mathrm{~cm}$; y la tercera en la floración. 
Se realizaron 2 deshierbes con machete aproximadamente a los 30 y 60 ddsm.

\section{Producción de biomasa y suministro de $\mathbf{N}$ de leguminosas}

En cada una de las unidades experimentales se trazaron 3 cuadrantes de $1 \mathrm{~m}^{2}$ de superficie (Mueller-Dombois y Ellemberg 1974), en los cuales se realizaron 2 muestreos de leguminosas. El primer muestreo fue realizado en la etapa de floración de leguminosas, aproximadamente a los 52 dds para el frijol común y a los 110 dds para los frijoles nescafé y arroz. El segundo muestreo fue realizado previo a la cosecha del grano de frijol común y a la rozadura de los frijoles nescafé y arroz. Se recolectó una mata completa (raíz y follaje) de cada leguminosa en floración, las raíces fueron colocadas en frascos de PVC de 1 $\mathrm{L}$, con $10 \%$ del volumen ocupado por acetileno, y se incubaron durante una hora para medir la actividad reductora de acetileno (Hardy et ál. 1968). Se extrajo una alícuota de $5 \mathrm{ml} \mathrm{y} \mathrm{se}$ conservó en tubos Vacutainer al vacío para un posterior análisis por cromatografía de gases del etileno producido. El análisis de cromatografía de gases se realizó en el CINVESTAV-IPN unidad Zacatenco, con un cromatógrafo Varian CP 3380 con detector de ionización de flama y columna Carbowax 1540/Porapak Q de $1.80 \mathrm{~m}$ x $4.6 \mathrm{~mm}$ DI. En ambos muestreos, el material vegetal fue llevado al laboratorio en donde se midió el peso seco del follaje, la raíz y los nódulos radicales, así como el número de nódulos. El peso seco se obtuvo con una balanza digital luego que el material fue deshidratado en horno a $65^{\circ} \mathrm{C}$ durante $72 \mathrm{~h}$. El follaje seco fue molido en un molino Willey (malla de $0,5 \mathrm{~mm}$ ) y se determinó la concentración de $\mathrm{N}$ mediante el método semi-microKjeldahl NOM-021-RECNAT-2000 (SEMARNAT 2002).

\section{Producción de biomasa y extracción de $\mathbf{N}$ del maíz}

En la madurez fisiológica se recolectaron al azar 5 matas de maíz bajo competencia completa en la parte central de cada una de las unidades experimentales. La biomasa aérea recolectada fue trasladada al laboratorio en donde se separó el rastrojo (hoja, caña y espiga), la bráctea (totomoxtle), el olote y el grano. Se midió el peso seco y la concentración de $\mathrm{N}$ de una manera similar como se hizo con las leguminosas.

\section{Balance parcial de nitrógeno}

El balance parcial de $\mathrm{N}$ se obtuvo mediante el cálculo de la diferencia entre la extracción de $\mathrm{N}$ (demanda) del cultivo de maíz y el contenido de $\mathrm{N}$ (suministro) en la biomasa de leguminosas (Rodríguez 1987, 1993, Volke et ál. 1998). En dicho cálculo se consideró que toda la biomasa de la leguminosa sería incorporada en el sitio donde se produjo, en el cual a través de la mineralización el $\mathrm{N}$ estaría disponible para el cultivo subsecuente. Asimismo, se asume una incorporación total del $\mathrm{N}$, y no se considera la tasa de descomposición de la biomasa ni las posibles pérdidas que podrían ocurrir por lixiviación y/o desnitrificación a través del tiempo. El suministro de $\mathrm{N}$ de leguminosa (SNL) se calculó como $\mathrm{SNL}=\Sigma[(\mathrm{kg}$ follaje $*$ concentración de $\mathrm{N})+(\mathrm{kg}$ raíz * concentración de $\mathrm{N}$ )]. La extracción de $\mathrm{N}$ por el maíz (ENM) se calculó a partir de la producción de biomasa y la concentración de $\mathrm{N}$ de la siguiente manera: $\mathrm{ENM}=\Sigma[(\mathrm{kg}$ rastrojo * concentración de $\mathrm{N})+(\mathrm{kg}$ bráctea $*$ concentración de $\mathrm{N})+(\mathrm{kg}$ olote $*$ concentración de $\mathrm{N})+$ $(\mathrm{kg}$ grano $*$ concentración de $\mathrm{N})]$. Debido a que no se recolectaron las raíces del maíz, la extracción de $\mathrm{N}$ por éstas se estimó al considerar una producción de raíces del 15\% de la producción de grano y rastrojo, y un contenido medio de $0,7 \%$ de N (Rodríguez 1993). Con base en la densidad de siembra los datos fueron expresados en kg.ha-1.

\section{Análisis estadístico}

Los datos se examinaron mediante un análisis de varianza (ANDEVA) para un diseño de bloques completos al azar y se hizo una comparación de medias de los tratamientos mediante la prueba de Tukey $(\mathrm{p} \leq 0,05)$ con SPSS v15.0. 


\section{RESULTADOS Y DISCUSIÓN}

\section{Producción de biomasa y suministro de $\mathbf{N}$ de leguminosas}

En la floración de las leguminosas, tanto el peso seco del follaje como el de la raíz mostraron una diferencia estadísticamente significativa entre tratamientos $(\mathrm{p}<0,01)$. El peso seco del follaje fue 7,5 veces más alto en FN que FC, y FA ocupó un lugar intermedio; mientras que el peso seco de la raíz fue 3,5 veces mayor en FN que FC, y de nuevo FA ocupó un lugar intermedio (Cuadro 2). El número de nódulos radicales fue de 46 a 63 por planta y no varió significativamente entre leguminosas $(\mathrm{p}=0,228)$; sin embargo, el peso seco de los nódulos fue significativamente $(\mathrm{p}<0,01)$ más alto en FN que FA y FC. Por el contrario, la medición de la actividad reductora de acetileno (ARA) mostró que FC tuvo 7 veces mayor actividad que FN y FA (Cuadro 2).

Cuadro 2. Producción de biomasa seca, nodulación y actividad reductora de acetileno (ARA) de las leguminosas en floración.

\begin{tabular}{|c|c|c|c|c|c|}
\hline Leguminosa & $\begin{array}{l}\text { Follaje } \\
\left(\text { g.mata }^{-1}\right)\end{array}$ & $\begin{array}{c}\text { Raíz } \\
\left(\text { g.mata }{ }^{-1}\right)\end{array}$ & $\begin{array}{l}\text { Nódulos } \\
\left(\text { n.mata }{ }^{-1}\right)\end{array}$ & $\begin{array}{l}\text { Nódulos } \\
\left(\text { g.mata }{ }^{-1}\right)\end{array}$ & $\begin{array}{l}\text { ARA -etileno- } \\
\left(\mathrm{ml} .100 \mathrm{ml}^{-1}\right)\end{array}$ \\
\hline Frijol (FC) & $4,6 \mathrm{c}^{\mathrm{g}}$ & $0,68 \mathrm{~b}$ & $56,8 \mathrm{a}$ & $0,06 \mathrm{~b}$ & $4,024 \mathrm{a}$ \\
\hline Nescafé (FN) & 34,3 a & $2,36 \mathrm{a}$ & $46,4 \mathrm{a}$ & $0,09 \mathrm{a}$ & $0,568 \mathrm{~b}$ \\
\hline Arroz (FA) & $10,1 \mathrm{~b}$ & $1,73 \mathrm{a}$ & $63,1 \mathrm{a}$ & $0,03 \mathrm{c}$ & $0,307 \mathrm{~b}$ \\
\hline
\end{tabular}

I Medias con distinta letra en una columna son significativamente diferentes (Tukey $\mathrm{p} \leq 0,05$ ).

La mayor producción de biomasa de FN se debe posiblemente a la expresión de su fenología, ya que ésta leguminosa se caracteriza por un crecimiento vigoroso, con alta producción de biomasa y excelente cobertura del suelo (CIDICCO 1991, Buckles y Triomphe 1999); en cambio FC tiene un ciclo de crecimiento corto y produce menor cantidad de biomasa debido a la producción de granos comestibles que constituyen un complemento nutricional indispensable de la alimentación familiar (Acuña y Uribe 1996). Además, es importante señalar que no obstante al momento de la evaluación todas las leguminosas se encontraban en floración, FC tenía solo 52 dds mientras FN y FA 110 dds.

Es difícil conocer la fracción de $\mathrm{N}$ del tejido de las leguminosas que ha sido fijado biológicamente de la atmosfera, debido a que ningún método es preciso y totalmente confiable para su medición (Valles de la Mora et ál. 2003); sin embargo, la medición de ARA proporciona un índice de la actividad fijadora del nitrógeno, y es en la etapa de floración en donde se presenta una mayor ARA debido a un mayor flujo de carbono hacia la simbiosis mutualista Rhizobiumleguminosa (Hardy et ál. 1968). Así, la mayor ARA de FC indica que esta especie estableció una simbiosis mutualista más eficiente en comparación con las otras 2 leguminosas, lo cual se debió posiblemente a la presencia de cepas nativas de Rhizobium, el genotipo de la planta y las condiciones ambientales, ya que la FBN está determinada por la interacción de estos factores (Acuña y Uribe 1996).

Al momento de la cosecha de FC y la rozadura de FN y FA, la cantidad de materia seca (MS) de raíz y de follaje fue significativamente más alta $(\mathrm{p}<0,01)$ en FN y FA que en FC (Cuadro 3). La concentración de $\mathrm{N}$ en el follaje varió significativamente $(p<0,01)$ entre leguminosas, con los valores más altos en FN y FA que en FC; mientras que en la raíz no hubo una diferencia estadística significativa en la concentración de $\mathrm{N}$ entre leguminosas $(\mathrm{p}=0,392)$ (Figura 1). 
Cuadro 3. Producción de biomasa seca (MS) y contenido de N del follaje y la raíz de las leguminosas en la cosecha.

\begin{tabular}{lcccc}
\hline Leguminosas & Raíz & Follaje & Raíz & \multicolumn{2}{c}{ Follaje } \\
& \multicolumn{2}{c}{ MS $\left(\mathrm{kg} \cdot h a^{-1}\right)$} & & $\mathrm{N}\left(\mathrm{kg} \cdot h a^{-1}\right)$ \\
\hline Frijol (FC) & $54,0 \mathrm{~b}^{\mathrm{g}}$ & $415,5 \mathrm{~b}$ & $0,67 \mathrm{~b}$ & $8,2 \mathrm{c}$ \\
Nescafé (FN) & $131,0 \mathrm{a}$ & $1008,0 \mathrm{a}$ & $1,29 \mathrm{a}$ & $24,4 \mathrm{~b}$ \\
Arroz (FA) & $148,4 \mathrm{a}$ & $1141,7 \mathrm{a}$ & $1,26 \mathrm{a}$ & $34,1 \mathrm{a}$ \\
\hline
\end{tabular}

${ }^{9}$ Medias con distinta letra en una columna son significativamente diferentes (Tukey $p \leq 0,05$ ).

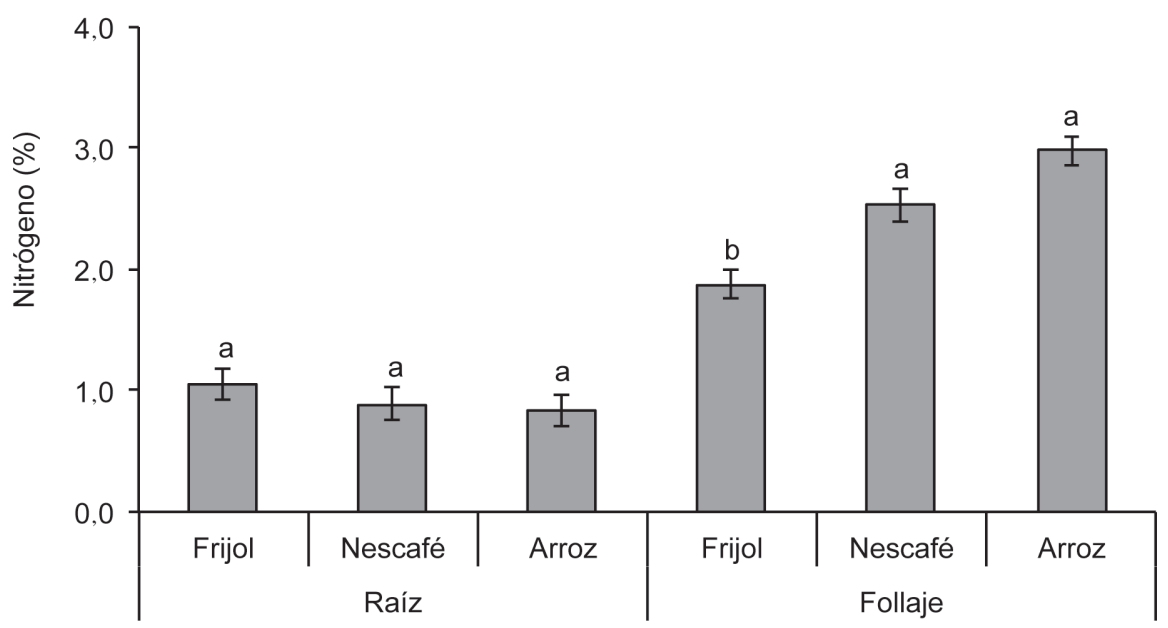

Fig. 1. Concentración de $\mathrm{N}$ en raíz y follaje de las leguminosas. Las barras con distinta letra dentro de raíz y follaje son significativamente diferentes (Tukey $\mathrm{p} \leq 0,05$ ).

La menor concentración de $\mathrm{N}$ en el follaje de FC que en las otras leguminosas, no obstante la mayor ARA observada en FC en la etapa de floración, se relaciona posiblemente con la movilización del elemento hacia las vainas y la formación del grano (Da Silva et ál. 1993). La biomasa producida por FN y FA fue de 1139,0 y 1290,1 kg.ha-1 MS, respectivamente. Estos valores son intermedios a los obtenidos en Yucatán por Ayala et ál. (2009) y Castillo et ál. (2010), en los cuales Mucuna produjo 1950 y 734 kg.ha- ${ }^{-1}$ MS, respectivamente. En función de la producción de MS y la concentración de $\mathrm{N}$, el aporte de las leguminosas fue en promedio de 8,9; 25,7 y 35,4 kg.ha- ${ }^{-1}$ de $\mathrm{N}$ para FC, FN y FA, respectivamente. El FN y FA tuvieron un contenido similar de $\mathrm{N}$ en raíz y fue casi el doble al de FC; mientras que en follaje el $\mathrm{N}$ fue significativamente $(\mathrm{p}<0,01)$ más alto en $\mathrm{FA}$, seguido por FN y FC (Cuadro 3).

\section{Producción de biomasa y extracción de $\mathbf{N}$ del maíz}

Al momento de la cosecha no hubo una diferencia estadística significativa entre tratamientos $(p=0,721)$ en la cantidad de MS de las diferentes estructuras en que fue dividida la planta de maíz (raíz, rastrojo, bráctea, olote y grano; Cuadro 4). El rendimiento de grano de maíz fue en promedio de 2390,5 kg.ha-1. La ausencia de 
Cuadro 4. Extracción de nitrógeno por las diferentes estructuras de la planta de maíz.

\begin{tabular}{|c|c|c|c|c|c|c|c|c|c|c|}
\hline \multirow{2}{*}{ Tratamiento } & \multicolumn{5}{|c|}{ Materia seca del maíz (kg.ha-1) } & \multicolumn{5}{|c|}{ Nitrógeno extraído por el maíz (kg.ha-1) } \\
\hline & Raíz \& & Rastrojo & Bráctea & Olote & Grano & Raíz \& & Rastrojo & Bráctea & Olote & Grano \\
\hline Testigo & $1048 \mathrm{a}^{\mathrm{J}}$ & 3310 a & $784 \mathrm{a}$ & $568 \mathrm{a}$ & $2345 \mathrm{a}$ & $7,3 \mathrm{a}$ & $9,6 \mathrm{a}$ & $2,1 \mathrm{a}$ & $1,5 \mathrm{a}$ & $20,5 \mathrm{a}$ \\
\hline Frijol (FC) & $1031 \mathrm{a}$ & $3010 \mathrm{a}$ & $784 \mathrm{a}$ & $600 \mathrm{a}$ & $2540 \mathrm{a}$ & $7,2 \mathrm{a}$ & $8,7 \mathrm{a}$ & $2,1 \mathrm{a}$ & $1,6 \mathrm{a}$ & $22,3 \mathrm{a}$ \\
\hline Nescafé (FN) & $1101 \mathrm{a}$ & $3518 \mathrm{a}$ & $782 \mathrm{a}$ & $540 \mathrm{a}$ & $2535 \mathrm{a}$ & 7,7 a & $10,2 \mathrm{a}$ & $2,1 \mathrm{a}$ & $1,4 \mathrm{a}$ & $22,2 \mathrm{a}$ \\
\hline Arroz (FA) & 958 a & 3133 a & $628 \mathrm{a}$ & $487 \mathrm{a}$ & $2142 \mathrm{a}$ & $6,7 \mathrm{a}$ & $9,0 \mathrm{a}$ & $1,7 \mathrm{a}$ & $1,3 \mathrm{a}$ & $18,8 \mathrm{a}$ \\
\hline
\end{tabular}

\& Estimado con base en Rodríguez (1993). ${ }^{\mathbb{9}}$ Medias con distinta letra en una columna son significativamente diferentes (Tukey $\mathrm{p} \leq 0,05)$.

efecto de la siembra de $\mathrm{CC}$ en el rendimiento de maíz ha sido observada en otros trabajos (Pool et ál. 1997, Castillo et ál. 2010). La planta de maíz utilizó una mayor cantidad de $\mathrm{N}$ para la producción de grano, y éste se diferenció estadísticamente $(\mathrm{p}<0,001)$ del rastrojo, las brácteas $\mathrm{y}$ el olote, los cuales demandaron $\mathrm{N}$ en menor proporción (Figura 2). La mayor extracción de $\mathrm{N}$ se presentó en el grano, seguido por rastrojo, raíz, bráctea y olote, con 20,9; 9,4; 7,2; 2,0 y 1,5 kg.ha ${ }^{-1} \mathrm{~N}$, respectivamente (Cuadro 4). La planta de maíz tuvo en promedio una tasa de extracción de $17,2 \mathrm{~kg} \cdot \mathrm{ha}^{-1}$ de $\mathrm{N}$ por cada tonelada de grano.
Castillo et ál. (2010) encontraron que el maíz en asociación con FN tuvo un rendimiento de grano de $843 \mathrm{~kg} \cdot \mathrm{ha}^{-1}$ y extrajo 11,5; 0,85 y 6,85 kg.ha-1 de $\mathrm{N}$ en grano, olote y rastrojo, respectivamente. El nivel de rendimiento y de extracción de $\mathrm{N}$ que fue obtenido en el presente trabajo se relaciona posiblemente con las características de fertilidad del suelo en las que fue establecido el experimento (Cuadro 1). Asimismo, la aplicación del biofertilizante foliar y la inoculación de micorrizas arbusculares en todos los tratamientos, pudo haber favorecido el crecimiento y el rendimiento del maíz.

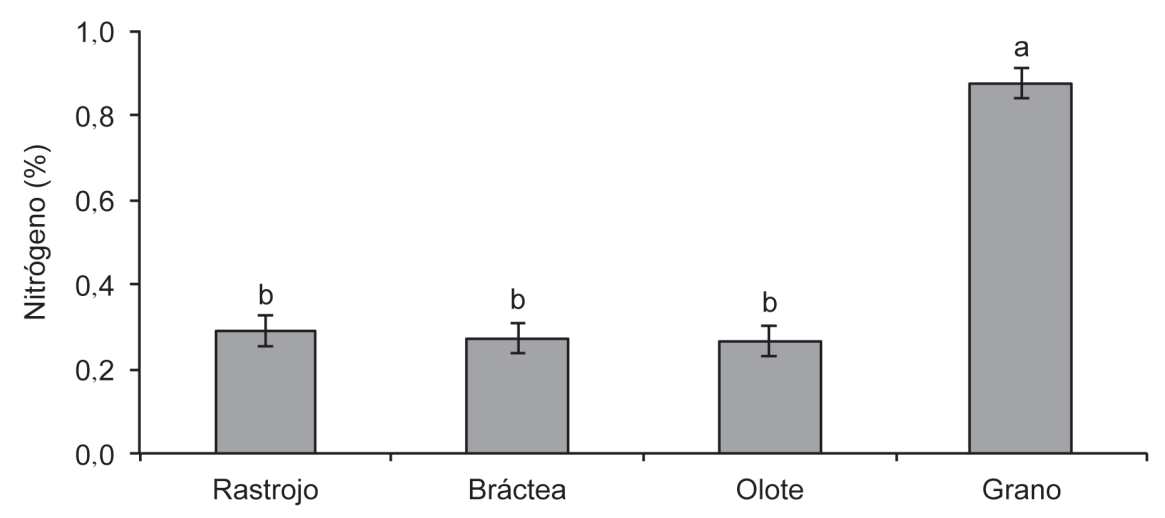

Fig. 2. Concentración de nitrógeno en diferentes estructuras de la planta de maíz. Las barras con distinta letra son significativamente diferentes (Tukey $\mathrm{p} \leq 0,05$ ). 


\section{Balance parcial de nitrógeno}

El balance parcial obtenido como la diferencia de la cantidad de $\mathrm{N}$ extraído por el maíz (Cuadro 4) y el contenido de $\mathrm{N}$ de las leguminosas (Cuadro 3), mostró que el testigo y FC no alcanzaron a satisfacer la extracción del grano de maíz, por lo tanto, el balance fue negativo. Por el contrario, FN y FA presentaron un balance positivo en relación con la extracción de $\mathrm{N}$ del grano o la mazorca de maíz, pero si se considera la extracción total de la planta de maíz (raíz, rastrojo y mazorca) el balance es negativo en todos los tratamientos (Cuadro 5). Lo anterior indica que FA podría cubrir la extracción de $\mathrm{N}$ de la parte aérea del cultivo de maíz (mazorca y rastrojo), pero es insuficiente si además se considera la extracción de la raíz; FN cubriría la extracción de $\mathrm{N}$ de la mazorca, y FC no alcanzaría a cubrir la extracción de $\mathrm{N}$ del grano. En la práctica común de los productores no se extrae la raíz de la planta, y ocasionalmente se extrae el rastrojo para la alimentación del ganado, lo más frecuente es la cosecha de la mazorca.
Por lo tanto, FA mostró un mejor desempeño que FN y FC para cubrir la extracción de $\mathrm{N}$ por la cosecha del cultivo de maíz en las parcelas estudiadas. No obstante, es de hacer notar que el $\mathrm{N}$ del follaje de las leguminosas difícilmente estará disponible en su totalidad en el siguiente ciclo de cultivo; se ha observado que solo el $65 \%$ del follaje de Mucuna se descompone en un ciclo de cultivo (Cruz et ál. 2002). Asimismo, es evidente que en el tratamiento testigo se presentaría un déficit del nutrimento, por lo cual, si no existe una adecuada reposición de $\mathrm{N}$, cuestiona la sustentabilidad del monocultivo de maíz. Un balance negativo conduciría gradualmente a reducir la capacidad productiva del suelo y afectaría el rendimiento y la rentabilidad de las actividades agrícolas (Flores y Sarandón 2002). De acuerdo con Ahiabor et ál. (2007) una forma de mantener y/o mejorar el contenido de $\mathrm{N}$ del suelo y la productividad de los cultivos es la siembra de leguminosas como CC en un sistema de rotación que permita retener los residuos hacia la siguiente temporada de cultivo.

Cuadro 5. Balance parcial de nitrógeno (kg.ha $\left.{ }^{-1}\right)$ de los tratamientos evaluados.

\begin{tabular}{lccc}
\hline Tratamiento & \multicolumn{3}{c}{ Estructuras de la planta de maíz } \\
\cline { 2 - 4 } & Grano & Mazorca & Total (planta completa de maíz) \\
\hline Testigo & $-20,5$ & $-24,2$ & $-41,1$ \\
Frijol (FC) & $-12,8$ & $-16,5$ & $-32,4$ \\
Nescafé (FN) & 4,4 & 0,8 & $-17,0$ \\
Arroz (FA) & 16,5 & 13,5 & $-2,3$ \\
\hline
\end{tabular}

IMazorca=bráctea + olote + grano.

\section{CONCLUSIÓN}

La producción de biomasa seca fue mayor en los frijoles arroz y nescafé (1290 y 1139 $\left.\mathrm{kg} \cdot \mathrm{ha}^{-1}\right)$ que en frijol común (470 kg.ha-1), con un contenido total de $\mathrm{N}$ de 35,$4 ; 25,7$ y $8,9 \mathrm{~kg} \mathrm{ha}^{-1}$ $\mathrm{N}$, respectivamente. El rendimiento de grano de maíz fue $2390,5 \mathrm{~kg}$.ha-1; la extracción de $\mathrm{N}$ fue mayor en el grano, seguido de rastrojo, raíz, bráctea y olote, con 20,9; 9,4; 7,2; 2,0 y 1,5 kg.ha ${ }^{-1}$
$\mathrm{N}$, respectivamente. Los frijoles arroz y nescafé podrían compensar la extracción de $\mathrm{N}$ del grano o la mazorca del maíz; sin embargo, si se considera la extracción de toda la planta el balance es negativo en todos los tratamientos. Estos resultados indican la importancia de incorporar leguminosas como cultivo de cobertura (CC) y retener sus residuos para contribuir paulatinamente a un balance positivo de $\mathrm{N}$ en el cultivo de maíz. 


\section{LITERATURA CITADA}

ACUÑA O., URIBE L. 1996. Inoculación del frijol común con tres cepas seleccionadas de Rhizobium leguminosarum bv. phaseoli. Agronomía Mesoamericana 7(1):35-40.

AHIABOR B.D.K., FOSU M., TIBO I., SUMAILA I. 2007. Comparative nitrogen fixation, native arbuscular mycorrhiza formation and biomass production potentials of some grain legume species grown in the field in the Guinea savanna zone of Ghana. West African Journal of Applied Ecology 11:72-80.

AYALA A., KRISHNAMURTHY L., BASALTO G.J.A. 2009. Leguminosas de cobertura para mejorar y sostener la productividad de maíz en el sur de Yucatán. Terra Latinoamericana 27:63-69.

BECKER M., JOHNSON D.E. 1998. Legumes as dry season fallow in upland rice-based systems of West Africa. Biology and Fertility of Soils 27:358-367.

BUCKLES D., TRIOMPHE B. 1999. Adoption of mucuna in the farming system of northern Honduras. Agroforestry Systems 47:67-91.

CASTELLANOS R.J.Z., CUETO W.J., MACÍAS C.M.C., SALINAS G.J.R., TAPIA V.L.M., CORTÉS J.J.M., GONZÁLEZ A.I.J., MATA V.H., MORA G.M., VÁSQUEZ H.A., VALENZUELA S.C., ENRÍQUEZ R.S.A. 2005. La fertilización en los cultivos de maíz, sorgo y trigo en México. Instituto Nacional de Investigaciones Forestales, Agrícolas y Pecuarias. Guanajuato, México. 44 p.

CASTILLO J.B., CAAMAL J.A., JIMÉNEZ J.J., BAUTISTA M.F., AMAYA M.J., RODRÍGUEZ R. 2010. Evaluación de tres leguminosas como coberturas asociadas con maíz en el trópico subhúmedo. Agronomía Mesoamericana 21(1):39-50.

CIDICCO (CENTRO INTERNACIONAL DE INFORMACIÓN SOBRE CULTIVOS DE COBERTURA). 1991. El uso del frijol terciopelo (Mucuna pruriens) en Honduras, para control de malezas y asociación con maíz. Noticias sobre el uso de los cultivos de cobertura. Tegucigalpa, Honduras. 4 p.

CRUZ A.G., SALGADO S., CATZIN F.J., ORTIZ A.I. 2002. Descomposición del follaje de Nescafé (Mucuna spp.) en la época seca. Interciencia 27(11):625-630.

DA SILVA P.M., TSAI S.M., BONETTI R. 1993. Response to inoculation and $\mathrm{N}$ fertilization for increased yield and biological nitrogen fixation of common bean (Phaseolus vulgaris L.). Plant and Soil 152:123-130.

DECHERT G., VELDKAMP E., BRUMME R. 2005. Are partial nutrient balances suitable to evaluate nutrient sustainability of land use systems? Results from a case study in Central Sulawesi, Indonesia. Nutrient Cycling in Agroecosystems 72:201-212.

FAO (FOOD AND AGRICULTURE ORGANIZATION OF THE UNITED NATIONS). 1999. Guía para el manejo eficiente de la nutrición de las plantas. Organización de las Naciones Unidas para la Agricultura y la Alimentación, Roma. 20 p.

FLORES C., SARANDÓN S.J. 2002. ¿Racionalidad económica versus sustentabilidad ecológica? El ejemplo del costo oculto de la pérdida de fertilidad del suelo durante el proceso de Agriculturización en la Región Pampeana Argentina. Revista de la Facultad de Agronomía, La Plata 105(1):52-67.

FLORES M.C.M., MADRIZ I.P.M., WARNOCK D.E., PARRA R., TRUJILLO D.E., LEAL A. 2005. Evaluación de altura de plantas y componentes del rendimiento de seis genotipos del género Vigna en dos localidades de Venezuela. Revista de la Facultad de Agronomía (LUZ) 22:354-368.

FRANKE A.C., LABERGE G., OYEWOLE B.D., SCHULZ S. 2008. A comparison between legume technologies and fallow, and their effects on maize and soil traits, in two distinct environments of the West African savannah. Nutrient Cycling in Agroecosystems $82: 117-135$.

HARDY R.W.F., HOLSTEN R.D., JACKSON E.K., BURNS R.C. 1968. The acetylene-ethylene assay for $\mathrm{N}_{2}$ fixation: Laboratory and Field Evaluation. Plant Physiology 43:1185-1207.

HOUNGNANDAN P., SANGINGA N., WOOMER P., VANLAUWE B., VAN CLEEMPU O. 2000. Response of Mucuna pruriens to symbiotic nitrogen fixation by rhizobia following inoculation in farmers fields in the derived savanna of Benin. Biology and Fertility of Soils 30:558-565.

HUNGRIA M., CAMPO R.J., MENDES I.C. 2003. Benefits of inoculation of the common bean (Phaseolus vulgaris) crop with efficient and competitive Rhizobium tropici strains. Biology and Fertility of Soils 39:88-93.

IUSS (INTERNATIONAL UNION OF SOIL SCIENCES) Grupo de Trabajo WRB. 2007. Base Referencial Mundial del Recurso Suelo. Primera actualización 2007. Informes sobre Recursos Mundiales de Suelos No. 103. FAO, Roma. 117 p.

LANDON J.R. 1991. Booker Tropical Soil Manual. Booker Tate, EUA. 474 p.

MENDOZA M., MOSQUEDA C.J., RANGEL A., LÓPEZ A., RODRÍGUEZ S.A., LATOURNERIE L., MORENO E. 2006. Densidad de población y fertilización nitrogenada en la clorofila, materia seca y rendimiento de maíz Normal y QPM. Agricultura Técnica en México 32(1):89-99.

MUELLER D., ELLENBERG H. 1974. Aims and methods of vegetation ecology. Wiley. New York. 547 p.

NOORDWIJK M., SITOMPUL S.M., HAIRIAH K., LISTGARIUM E. 1995. Nitrogen supply from rotational or spatially zoned inclusion of leguminosae for sustainable maize production on an acid soil in Indonesia, pp. 779-784. In: R.A. Date (ed.). 
Plant interactions at low $\mathrm{pH}$. Kluwer Academic Publications, The Netherlands.

NYAMBATIE.M. 2002. Management and nutritive evaluation of Mucuna pruriens and Lablab purpureusmaize intercrops in the sub-humid highlands of northwestern Kenya. Ph. D. Dissertation. University of Florida, Gainesville, FL, USA. 234 p.

PÉREZ O.M.A., ETCHEVERS B.J.D., NAVARRO G.H., NÚÑEZ E.R. 2000. Aporte de los residuos del cultivo anterior al reservorio de nitrógeno en tepetates. Agrociencia 34(2):115-125.

POOL N.L., LEÓN M.N.S., GONZÁLEZ S.C.Y., FIGUEROA F.P. 1997. Frijol terciopelo, cultivo de cobertura en la agricultura chol del Valle de Tulijá, Chiapas, México. Terra 16(4):359-369.

RODRÍGUEZ S.J. 1987. Desarrollo de normas de fertilización para el cultivo de maíz y de cebada en el estado de Tlaxcala. Colegio de Postgraduados, Montecillo, estado de México. 162 p.

RODRÍGUEZ S.J. 1993. La fertilización de los cultivos: un método racional. Facultad de Agronomía, Pontificia Universidad Católica, Santiago, Chile. 291 p.
SEMARNAT. 2002. Norma Oficial Mexicana NOM-021RECNAT-2000, Que establece las especificaciones de fertilidad, salinidad y clasificación de suelos. Estudios, muestreo y análisis. Secretaría del Medio Ambiente y Recursos Naturales. Diario Oficial de la Federación. 2a Sección, 31 de diciembre de 2002.

VALLES DE LA MORA B., CADISCH G., ALUJASCHUNEMANN A. 2003. Comparación de metodologías de isótopos para evaluar fijación de $\mathrm{N}$ atmosférico y su destino en suelos y plantas. Agrociencia 37(2):117-129.

VAN DER POOL F. 1992. Soil mining: an unseen contributor to farm income in southern Mali. Royal Tropical Institute, Bulletin 325, Amsterdam, The Netherland, $47 \mathrm{p}$.

VOLKE H.V., ETCHEVERS B.J.D., SANJUAN R.A., SILVA P.T. 1998. Modelo de balance nutrimental para la generación de recomendaciones de fertilización para cultivos. Terra Latinoamericana 16:79-91. 
